\title{
ANÁLISE DIALÉLICA EM CLONES DE CANA-DE-AÇÚCAR ${ }^{(1)}$
}

\author{
IRLANE TOLEDO BASTOS ${ }^{(2,7)}$; MÁRCIO HENRIQUE PEREIRA BARBOSA ${ }^{(3,6)}$; \\ COSME DAMIÃO CRUZ ${ }^{(4,6)}$; WILLIAM LEE BURNQUIST ${ }^{(5)}$; \\ JOSÉ ANTÔNIO BRESSIANI ${ }^{(5)}$;FELIPE LOPES DA SILVA ${ }^{(3)}$
}

\begin{abstract}
RESUMO
Visando avaliar a ação gênica predominante em caracteres de importância econômica da cana-deaçúcar e identificar cruzamentos com potencial genético favorável para o desenvolvimento de novos cultivares, avaliaram-se oito genótipos segundo um esquema em dialelo desbalanceado, e também, progênies de 44 cruzamentos no delineamento látice $7 \times 7$, com três repetições, no Centro de Pesquisa e Melhoramento da Cana-de-Açúcar (CECA), da Universidade Federal de Viçosa. Os caracteres estudados foram: Brix \% caldo da cana, número de colmos, massa média de colmo, toneladas de colmos por hectare, toneladas de Brix por hectare e porcentagem de florescimento. Os resultados evidenciaram a importância tanto dos efeitos gênicos aditivos como dos não-aditivos na expressão das características avaliadas. Os genótipos SP82-6108 e IAC86-2210 e as combinações híbridas SP82-6108 x SP88-754, SP826108 x SP87-365, SP81-1763 x SP82-6108, IAC86-2210 x SP88-754, IAC86-2210 x SP86-96, IAC86-2210 x SP81-1763, SP88-819 x SP87-365, SP84-2029 x SP87-365 e SP88-754 x SP87-365, mostraram maior potencial para elevar a produtividade de cana-de-açúcar.
\end{abstract}

Palavras-chave: melhoramento genético, capacidade geral de combinação, capacidade específica de combinação.

\section{ABSTRACT \\ DIALLEL ANALYSIS OF SUGARCANE CLONES}

Aiming at the evaluation of the gene action predominant in sugarcane characters of economic importance and the identification of crossing with favorable genetic potential to the development of new cultivars, eight genotypes were evaluated according to the unbalanced diallel cross scheme. Progenies obtained from 44 crosses were evaluated on a $7 \times 7$ lattice experimental design with three replications at the Centro de Pesquisa e Melhoramento da Cana-de-açúcar (CECA) of the Universidade Federal de

$\left({ }^{1}\right)$ Parte da dissertação de Mestrado em Agronomia/Genética e Melhoramento de Plantas da primeira autora, apresentada à Universidade Federal de Viçosa (MG). Trabalho realizado com suporte financeiro e bolsa de estudo do CNPq. Recebido para publicação em 24 de setembro de 2002 e aceito em 17 de abril de 2003.

$\left({ }^{2}\right)$ Doutoranda em Genética e Melhoramento de Plantas, Departamento de Fitotecnia/Genética e Melhoramento de Plantas, Universidade federal de Viçosa, 36571-000, Viçosa (MG). E-mail: ibastos@alunos.ufv.br

$\left({ }^{3}\right)$ Departamento de Fitotecnia, Universidade federal de Viçosa. E-mail: barbosa@ufv.br

$\left({ }^{4}\right)$ Departamento de Biologia Geral, Universidade Federal de Viçosa. E-mail: cdcruz@mail.ufv.br

$\left(^{5}\right)$ Centro de Tecnologia Copersucar, Caixa Postal 162, 13400-970 Piracicaba (SP). E-mail: william@copersucar.com.br , bressiani@copersucar.com.br.

$\left({ }^{6}\right)$ Com bolsa de produtividade científica do CNPq.

$\left({ }^{7}\right)$ Bolsista da FAPEMIG. 


\begin{abstract}
Viçosa. The following characters were studied: juice Brix \%, stalk number, average stalk weight, tons of stalks per hectare, tons of Brix per hectare and flowering percent. The results emphasized the importance of either the additive and the non-additive genetic effects in the expression of those characteristics. The genotypes SP82-6108 and IAC86-2210 showed potential for increasing the sugarcane productivity, that is also valid for the following crossing combinations: SP82-6108 x SP88-754, SP82-6108 x SP87-365, SP811763 x SP82-6108, IAC86-2210 x SP88-754, IAC86-2210 x SP86-96, IAC86-2210 x SP81-1763, SP88-819 x SP87-365, SP84-2029 x SP87-365 and SP88-754 x SP87-365.
\end{abstract}

Key words: Genetic improvement, general combining ability, specific combining ability.

\section{INTRODUÇÃO}

Um dos principais desafios encontrados pelos melhoristas de plantas é a escolha das combinações parentais que vão gerar as populações segregantes a serem submetidas à seleção. Diversos métodos têm sido propostos para escolha de populações em espécies autógamas e alógamas (BAENZiger e Peterson, 1992). Dentre eles, destacam-se os cruzamentos dialélicos.

Em cana-de-açúcar, entretanto, os cruzamentos dialélicos têm apresentado algumas dificuldades, pois alguns clones apresentam incompatibilidade, quando cruzados e/ou são macho-estéreis. Outro problema é a ocorrência de autofecundação, uma vez que os métodos de emasculação artificial não são totalmente eficientes (Hogarth, 1980).

SPRAgUe e TAtum (1942) propuseram o uso dos cruzamentos dialélicos para estimar a capacidade geral de combinação (CGC) e a capacidade específica de combinação (CEC). A CGC refere-se à performance média de uma cultivar ou um clone em combinações híbridas ao passo que a CEC é usada para designar aqueles casos em que certas combinações híbridas são melhores ou piores que o esperado, com base na performance média de cultivares ou clones envolvidos. A CGC está associada a genes de efeitos principalmente aditivos, além dos efeitos epistáticos aditivos $\mathrm{x}$ aditivos. Por sua vez a CEC depende, basicamente, de genes com efeitos dominantes e de vários tipos de interações (CRUZ e RegazZI, 1994).

Estudos de genética quantitativa têm mostrado que a CGC é mais importante que a CEC para a maioria dos caracteres de importância econômica na cana-de-açúcar, como teor de açúcar, resistência a doenças tais como carvão, ferrugem e mal de Fiji; e conteúdo de fibra. A principal exceção é a tonelada de colmos por hectare, em que ambas as variâncias têm apresentado valores similares (HOGARTH, 1997).

Por outro lado, MilLer (1977) não encontrou diferença significativa da CGC para as características massa média de colmos, número de colmos, diâmetro de colmos, Brix, toneladas de colmos por hectare e toneladas de açúcar por hectare, enquanto a CEC foi significativa para todas essas características. Esse re- sultado é bastante incomum quando comparado a outros trabalhos (HogARTH, 1997; BRESSIANI et al, 2001). Talvez esses resultados possam ter ocorrido em conseqüência da autofecundação nos cruzamentos tendo em vista que Miller (1977) não utilizou nenhum método para preveni-la, conforme ressaltado por Hogarth (1980).

O fato de prevalecer a CGC para diversas características em cana-de-açúcar implica selecionar os genitores com base em sua performance. De outra forma, como a CEC tem-se mostrado tão importante quanto a CGC na expressão da característica toneladas de colmos por hectare, torna-se pouco provável predizer com precisão a performance dos cruzamentos. Nesse caso, grande número de cruzamentos deveria ser realizado e avaliado em experimentos com repetição para identificar as melhores famílias e prosseguir com a seleção em nível de indivíduos para obtenção dos clones.

O objetivo do presente trabalho foi avaliar a ação gênica predominante para algumas características e identificar cruzamentos com potencial genético desejável para o desenvolvimento de novas cultivares de cana-de-açúcar.

\section{MATERIAL E MÉTODOS}

Os genótipos IAC86-2210, SP81-1763, SP87365, SP82-6108, SP84-2029, SP86-96, SP88-754 e SP88-819 foram cruzados entre si a fim de obter os híbridos $\mathrm{F}_{1}$ 's e seus respectivos recíprocos, conforme o método 1 de Griffing para cruzamentos dialélicos (GRIFFING, 1956). Os cruzamentos foram realizados pela COPERSUCAR, em Camamu (BA). Para prevenir a autofecundação, todas as inflorescências fêmeas foram emasculadas usando tratamento com água quente (MACHADO JÚNIOR, et al., 1996).

As progênies obtidas foram plantadas no Centro de Pesquisa e Melhoramento da Cana-de-Açúcar (CECA) da Universidade Federal de Viçosa (MG), localizada no município de Oratórios (MG), com latitude $20^{\circ} 25^{\prime} \mathrm{S}$; longitude $42^{\circ} 48^{\prime} \mathrm{W}$; altitude $494 \mathrm{~m}$; solo LVE. 
Dos 56 possíveis cruzamentos e seus recíprocos, envolvendo oito genitores, apenas 44 produziram sementes suficientes para o experimento. Empregouse o delineamento em látice $7 \times 7$ com três repetições. Para compor os 49 tratamentos, acrescentaram-se cinco famílias que não fizeram parte da análise dialélica. A obtenção e o transplantio dos "seedlings" foram realizados conforme descrito por BARBOSA e Silveira (2000).

O experimento totalizou 147 parcelas, sendo cada parcela composta por seis sulcos espaçados de $1,40 \mathrm{~m}$. Cada sulco constituiu-se por sete plantas eqüidistantes $0,5 \mathrm{~m}$, sendo o comprimento de cada sulco de 3,5 m. Utilizou-se como bordadura, nas laterais do experimento, a variedade RB72454. A adubação empregada foi de $500 \mathrm{~kg} \cdot \mathrm{ha}^{-1}$ do formulado contendo $5 \%$ de $\mathrm{N}, 25 \%$ de $\mathrm{P}_{2} \mathrm{O}_{5}$ e $25 \%$ de $\mathrm{K}_{2} \mathrm{O}$.

O plantio ocorreu em fevereiro de 1999 e, em julho de 1999, todas as plantas foram cortadas manualmente com facão, submetendo-se os "seedlings" à seleção natural para capacidade de rebrota em condições ambientes desfavoráveis, isto é, época seca e fria. Em julho de 2000, fez-se a coleta dos dados em cana-soca.

Os caracteres avaliados foram: Brix refratométrico, tomando aleatoriamente um colmo por indivíduo, cuja amostragem realizou-se no quinto entrenó, contado da base do colmo para o ápice, com posterior transformação para Brix médio por parcela; número total de colmos por sulco; número de colmos floridos por sulco, posteriormente transformado para porcentagem de florescimento; massa de 10 colmos por sulco, amostrados aleatoriamente, com posterior transformação para massa média de colmo por parcela; e massa total dos colmos por sulco e por parcela, com posterior transformação para toneladas de colmos por hectare (TCH). A partir do produto entre o Brix e o TCH obteve-se a variável tonelada de Brix por hectare (TBH).

As médias ajustadas dos 27 híbridos $F_{1}$ 's e dos 17 híbridos recíprocos, obtidos por meio da análise de variância em látice, foram transformadas em uma única média, de modo que permita a análise dos dados em dialelo desbalanceado com avaliação apenas dos híbridos $F_{1}$ 's, excluindo-se as informações dos recíprocos e dos pais, empregando método descrito por Martins Filho et. al. (1992). Se um dado tratamento possuía apenas a média do híbrido $\mathrm{F}_{1}$, ou apenas a média do híbrido recíproco, então, a média utilizada na análise dialélica para aquele tratamento representava o $F_{1}$ com três repetições, independentemente de sua origem. Se o tratamento, porém, possuía ambas as médias, então, uma nova média era obtida por meio desses dois valores, a qual passou a constituir a média do híbrido $\mathrm{F}_{1}$ deste tratamento, que conseqüentemente era oriunda de seis repetições.
Assim, a análise dialélica realizou-se com 28 tratamentos e os efeitos foram considerados fixos. Todas as análises estatísticas foram feitas com o auxílio do programa computacional em genética e estatística, Genes (Cruz, 1997).

\section{RESULTADOS E DISCUSSÃO}

As médias ajustadas dos 28 tratamentos, ou híbridos $\mathrm{F}_{1}$ 's foram submetidas ao teste de Sсотт e KnOTT (1974), ao nível de 5\%. Para implementar o teste, utilizou-se como divisor, a média harmônica do número de repetições que deram origem à média de cada família. Os resultados estão apresentados no quadro 1. Os tratamentos diferiram em relação a todos os caracteres.

Em relação à massa média de colmos observou-se, especialmente, variabilidade mais acentuada como constatado pelo grande número de agrupamentos formados.

Para as características número de colmos, Brix, TCH, TBH e porcentagem de florescimento, as médias dos tratamentos foram mais homogêneas, com evidência de três grupos abrangendo maior número de tratamentos e um grupo, apenas um ou poucos tratamentos.

Considerando apenas as variáveis TCH e TBH, de grande importância no melhoramento da cana-deaçúcar, verifica-se que seis cruzamentos foram estatisticamente superiores aos demais: SP82-6108 x SP88-754, SP82-6108 x SP87-365, SP81-1763 x SP826108, SP82-6108 x SP88-819, IAC86-2210 x SP88-754 e SP88-819 x SP87-365.

Os resultados das análises de variância dialélica estão apresentados no quadro 2 . O teste $\mathrm{F}$ foi estatisticamente significativo tanto para a CGC quanto para a CEC, o que mostra a importância tanto dos efeitos gênicos aditivos como dos não-aditivos na expressão das características avaliadas.

Nota-se também no quadro 2 que o componente quadrático associado à CGC foi superior ao componente quadrático associado à CEC para as variáveis número de colmos, Brix e porcentagem de florescimento.

Tal situação evidencia a predominância dos efeitos gênicos aditivos na expressão dessas variáveis. Hogarth et al. (1981) e Bressiani et al. (2001) também verificaram que a CGC foi superior à CEC para Brix e número de colmos. Já LyRene (1977) verificou que a CGC foi 4,4 vezes superior à CEC para porcentagem de florescimento. 
Quadro 1. Médias das famílias para número de colmos (NC), massa média de colmo (MMC), Brix médio (Brix), toneladas de cana por hectare $(\mathrm{TCH})$, toneladas de Brix por hectare $(\mathrm{TBH})$ e porcentagem de florescimento $(\mathrm{F})$

\begin{tabular}{|c|c|c|c|c|c|c|}
\hline Cruzamentos & NC & MMC & $\begin{array}{c}\text { Brix \% } \\
\text { caldo da cana }\end{array}$ & $\mathrm{TCH}$ & ТВH & $\mathrm{F}$ \\
\hline & & $\mathrm{kg}$ & & - & - & $\%$ \\
\hline SP82-6108 x SP88-754 & $263,14 \mathrm{~b}$ & $1,16 \mathrm{c}$ & $22,14 \mathrm{~b}$ & $3,23 \mathrm{~d}$ & 117,87 a & 25,98 a \\
\hline SP82-6108 x SP87-365 & $258,86 \mathrm{~b}$ & 1,28 a & 22,80 a & 115,78 a & 26,28 a & $8,92 \mathrm{c}$ \\
\hline SP81-1763 x SP82-6108 & $268,16 \mathrm{~b}$ & $1,23 \mathrm{~b}$ & $22,05 \mathrm{~b}$ & 113,34 a & 25,03 a & $8,14 \mathrm{c}$ \\
\hline SP82-6108 x SP88-819 & $274,29 \mathrm{~b}$ & $1,14 \mathrm{c}$ & $22,48 \mathrm{~b}$ & 111,32 a & 25,13 a & $3,99 \mathrm{~d}$ \\
\hline IAC86-2210 x SP88-754 & $256,99 \mathrm{~b}$ & $1,16 \mathrm{c}$ & $22,60 \mathrm{~b}$ & 110,94 a & 24,92 a & $10,43 \mathrm{c}$ \\
\hline SP88-819 x SP87-365 & 311,47 a & $1,01 \mathrm{f}$ & $22,30 \mathrm{~b}$ & 109,28 a & 24,12 a & $2,74 \mathrm{~d}$ \\
\hline IAC86-2210 x SP86-96 & 247,22 c & $1,90 \mathrm{~d}$ & $22,20 \mathrm{~b}$ & 107,33 a & $23,59 \mathrm{~b}$ & $12,56 \mathrm{c}$ \\
\hline IAC86-2210 x SP81-1763 & $242,94 \mathrm{c}$ & $1,00 \mathrm{~d}$ & 22,74 a & $104,35 \mathrm{~b}$ & $23,59 \mathrm{~b}$ & 25,80 a \\
\hline SP84-2029 x SP87-365 & $275,61 \mathrm{~b}$ & $1,03 \mathrm{f}$ & $22,18 \mathrm{~b}$ & $103,57 \mathrm{~b}$ & $22,99 \mathrm{~b}$ & $8,44 \mathrm{c}$ \\
\hline SP84-2029 x SP88-819 & 314,62 a & $0,84 \mathrm{j}$ & $22,55 \mathrm{~b}$ & $102,87 \mathrm{~b}$ & $23,18 \mathrm{~b}$ & $5,98 \mathrm{~d}$ \\
\hline SP88-754 x SP87-365 & $268,10 \mathrm{~b}$ & $1,06 \mathrm{e}$ & $22,65 \mathrm{~b}$ & $100,31 \mathrm{~b}$ & $22,78 \mathrm{~b}$ & $9,27 \mathrm{c}$ \\
\hline SP81-1763 x SP88-819 & 299,23 a & $0,98 \mathrm{~g}$ & $22,28 \mathrm{~b}$ & $100,28 \mathrm{~b}$ & $22,20 \mathrm{~b}$ & $10,12 \mathrm{c}$ \\
\hline SP82-6108 x SP86-96 & $234,43 \mathrm{c}$ & $1,12 \mathrm{~d}$ & $20,90 \mathrm{~d}$ & $99,91 \mathrm{~b}$ & $20,81 \mathrm{~b}$ & $3,61 \mathrm{~d}$ \\
\hline SP84-2029 x SP86-96 & 291,15 a & $0,96 \mathrm{~g}$ & $21,58 \mathrm{c}$ & $98,41 \mathrm{~b}$ & $21,14 \mathrm{~b}$ & $9,07 \mathrm{c}$ \\
\hline IAC86-2210 x SP82-6108 & $215,37 \mathrm{~d}$ & $1,14 \mathrm{c}$ & 23,08 a & $96,75 \mathrm{~b}$ & $22,33 \mathrm{~b}$ & $7,17 \mathrm{~d}$ \\
\hline IAC86-2210 x SP84-2029 & $250,76 \mathrm{c}$ & $1,02 \mathrm{f}$ & $22,58 \mathrm{~b}$ & $95,22 \mathrm{~b}$ & $21,35 \mathrm{~b}$ & $6,82 \mathrm{~d}$ \\
\hline SP86-96 x SP88-819 & $279,24 \mathrm{~b}$ & $0,96 \mathrm{~g}$ & $21,01 \mathrm{~d}$ & $94,57 \mathrm{~b}$ & $19,67 \mathrm{c}$ & $11,89 \mathrm{c}$ \\
\hline SP82-6108 x SP84-2029 & $238,02 \mathrm{c}$ & $1,07 \mathrm{e}$ & $21,74 \mathrm{c}$ & $94,51 \mathrm{~b}$ & $20,47 \mathrm{c}$ & $4,40 \mathrm{~d}$ \\
\hline SP84-2029 x SP88-754 & $225,43 \mathrm{c}$ & $1,04 \mathrm{f}$ & $22,30 \mathrm{~b}$ & 89,65 c & $20,02 \mathrm{c}$ & $4,81 \mathrm{~d}$ \\
\hline SP81-1763 x SP87-365 & $246,99 \mathrm{c}$ & $0,95 \mathrm{~g}$ & $21,93 \mathrm{c}$ & $84,40 \mathrm{c}$ & $18,36 \mathrm{c}$ & $18,56 \mathrm{~b}$ \\
\hline SP88-754 x SP88-819 & $264,57 \mathrm{~b}$ & $0,86 \mathrm{i}$ & $22,10 \mathrm{~b}$ & $82,50 \mathrm{c}$ & $18,27 \mathrm{c}$ & $9,38 \mathrm{c}$ \\
\hline SP81-1763 x SP88-754 & $239,17 \mathrm{c}$ & $0,90 \mathrm{~h}$ & $22,40 \mathrm{~b}$ & $81,12 \mathrm{c}$ & $18,07 \mathrm{c}$ & $18,69 \mathrm{~b}$ \\
\hline SP86-96 x SP88-754 & $247,61 \mathrm{c}$ & $0,94 \mathrm{~g}$ & $21,57 \mathrm{c}$ & $79,67 \mathrm{c}$ & $17,33 \mathrm{c}$ & $9,06 \mathrm{c}$ \\
\hline IAC $86-2210$ x SP88-819 & $205,34 \mathrm{~d}$ & $1,05 \mathrm{e}$ & 23,69 a & $79,48 \mathrm{c}$ & $18,85 \mathrm{c}$ & $11,67 \mathrm{c}$ \\
\hline SP81-1763 x SP84-2029 & $242,06 \mathrm{c}$ & $0,91 \mathrm{~h}$ & $22,08 \mathrm{~b}$ & $78,99 \mathrm{c}$ & $17,43 \mathrm{c}$ & $14,50 \mathrm{~b}$ \\
\hline IAC86-2210 x SP87-365 & $203,52 \mathrm{~d}$ & $0,95 \mathrm{~g}$ & 22,99 a & $78,72 \mathrm{c}$ & $18,11 \mathrm{c}$ & $9,35 \mathrm{c}$ \\
\hline SP81-1763 x SP86-96 & $254,03 \mathrm{c}$ & $0,80 \mathrm{k}$ & $22,22 \mathrm{~b}$ & $78,63 \mathrm{c}$ & $17,46 \mathrm{c}$ & $18,51 \mathrm{~b}$ \\
\hline SP86-96 x SP87-365 & $196,88 \mathrm{~d}$ & 0,681 & $22,14 \mathrm{~b}$ & $52,00 \mathrm{~d}$ & $11,16 \mathrm{~d}$ & $5,80 \mathrm{~d}$ \\
\hline Média geral & 252,21 & 1,01 & 22,23 & 95,67 & 21,23 & 9,97 \\
\hline
\end{tabular}

As médias seguidas pela mesma letra constituem grupos homogêneos, segundo método de Scott Knott a 5\% de probabilidade.

Seus estudos revelaram que é mais freqüente a ocorrência de florescimento em populações derivadas do cruzamento entre clones que florescem com maior intensidade. Esse fato teria implicação direta na seleção, pois aumentaria o descarte de clones devido ao florescimento ser indesejável na maioria dos sistemas de produção de cana-de-açúcar.

Os efeitos gênicos não-aditivos foram predominantes para TCH e TBH. Tal fato é conseqüência dos maiores valores dos componentes quadráticos associado à CEC, para essas variáveis (Quadro 2). No entanto, alguns estudos de genética quantitativa (Yang e Chu, 1962; Hogarth, 1977 e Hogarth et al., 1981), realizados com cana-de-açúcar, mostraram que os efeitos gênicos aditivos e não-aditivos são igualmente importantes na expressão da TCH. Por outro lado, BRESSIANI et al. (2001) mostraram em seu trabalho que a CGC foi superior a CEC para TCH. 
Quadro 2. Resumo das análises de variância do dialelo desbalanceado e estimativas dos componentes quadráticos relativos aos efeitos da capacidade geral de combinação ( $\hat{\phi})_{\mathrm{g}}$ e específica de combinação ( $\left.\hat{\phi}_{\mathrm{s}}\right)$ para número de colmos (NC), massa média de colmo (MMC), Brix médio (Brix), toneladas de cana por hectare (TCH), toneladas de Brix por hectare $(\mathrm{TBH})$ e porcentagem de florescimento $(\mathrm{F})$

\begin{tabular}{|c|c|c|c|c|c|c|c|}
\hline \multirow{2}{*}{$\mathrm{FV}$} & \multirow{2}{*}{ GL } & \multicolumn{6}{|c|}{ Quadrados médios } \\
\hline & & NC & MMC & $\begin{array}{c}\text { Brix \% } \\
\text { caldo da cana }\end{array}$ & $\mathrm{TCH}$ & $\mathrm{TBH}$ & $\mathrm{F}$ \\
\hline & & \multicolumn{3}{|c|}{$\mathrm{kg}$} & \multicolumn{2}{|c|}{$\longrightarrow \mathrm{t} \longrightarrow$} & $\%$ \\
\hline Tratamentos & 27 & $3504,51^{* *}$ & $0,0858^{* *}$ & $1,66^{* *}$ & $1003,60^{* *}$ & $51,64^{* *}$ & $151,92^{* *}$ \\
\hline CGC & 7 & $5219,37^{* *}$ & $0,2158^{* *}$ & $4,59 * *$ & $1310,74^{* *}$ & $77,02^{* *}$ & $453,49^{* *}$ \\
\hline CEC & 20 & $2904,31^{* *}$ & $0,0403 * *$ & $0,64^{* *}$ & $896,10^{* *}$ & $42,76^{* *}$ & $46,37^{*}$ \\
\hline Resíduo & 78 & 632,80 & 0,000373 & 0,27 & 66,12 & 3,05 & 23,16 \\
\hline$\hat{\phi}_{g}$ & & 162,75 & 0,0076 & 0,15 & 44,16 & 2,62 & 15,27 \\
\hline$\hat{\phi}_{\mathrm{s}}$ & & 80,60 & 0,0085 & 0,08 & 176,71 & 8,45 & 1,64 \\
\hline $\mathrm{CV} \%$ & & 10,11 & 1,91 & 2,36 & 8,76 & 8,48 & 50,59 \\
\hline
\end{tabular}

*,** Significativo a $5 \%$ e $1 \%$ de probabilidade pelo teste $\mathrm{F}$ respectivamente.

Quanto a variável massa média de colmo, nota-se que as estimativas dos componentes quadráticos, associados à CGC e à CEC, foram equivalentes, indicando que ambos os efeitos, aditivos e não-aditivos, são igualmente importantes na expressão dessa característica. De outra forma, HogARTH (1977) verificou que a CGC foi mais importante que a CEC para a variável massa média de colmo.

As estimativa dos efeitos da capacidade geral de combinação $\left(\hat{\mathrm{g}}_{\mathrm{i}}\right)$ fornecem a potencialidade do genótipo em gerar boas populações para o melhoramento, e proporcionam informações sobre a concentração de alelos predominantemente aditivos em seus efeitos. Quanto mais altas forem as estimativas de $\hat{g}_{i}$, positivas ou negativas, o genitor em questão é muito superior ou inferior aos demais incluídos no dialelo, e quanto mais próximas de zero forem essas estimativas, o comportamento do genitor não difere da média geral dos cruzamentos em questão (Cruz e RegazZI, 1994).

As estimativas desses efeitos encontram-se no quadro 3. O genitor SP82-6108 apresentou valores elevados e positivos de $\hat{g}_{i}$ para as variáveis massa média de colmo, TCH e TBH. Para a variável número de colmos a estimativa de $\hat{g}_{i}$ não difere estatisticamente de zero. Tal fato implica a possível utilização deste genitor para formação de uma população-base, em que progênies mais produtivas, com maior diâmetro de colmos ( $\hat{\mathrm{g}}_{\mathrm{i}}$ positivo e elevado para massa média de colmo) e menor perfilhamento ( $\hat{g}_{i}$ não difere estatisticamente de zero para número de colmos), possam ser selecionadas. Para essas inferências, admitiu-se correlação negativa entre o número de colmos e a massa média de colmos, conforme KANG et al. (1983) e REDDY e REDDI (1986). É importante ressaltar que as variáveis altura de plantas e diâmetro de colmos não foram avaliadas neste experimento. Assim, as previsões sobre o diâmetro de colmos das progênies não são totalmente esclarecidas. Portanto, a maior ou menor massa de colmo poderá ser uma conseqüência da maior ou menor altura de plantas e não função do diâmetro de colmos.

O genitor IAC86-2210 destacou-se em elevar a TBH ( $g_{i}$, positivo e elevado). Foi o único genótipo a apresentar estimativa de $g_{i}$, para Brix, positiva e estatisticamente diferente de zero. As demais estimativas de $g_{i}$, para essa variável, foram negativas ou não diferiram estatisticamente de zero.

O genitor IAC86-2210 não é indicado para cruzamentos cujo objetivo é elevar a produtividade de cana, pois apresentou $\mathrm{g}_{\mathrm{i}}$ não diferente estatisticamente de zero para a variável TCH. Outro fator desfavorável desse genitor foi a alta porcentagem de florescimento em suas progênies ( $g_{i}$ positivo e elevado para a variável porcentagem de florescimento).

Os genitores SP84-2029 e SP88-819 apresentaram estimativas de $g_{i}$ elevadas e positivas para a variável número de colmos, e estimativas de $\mathrm{g}_{\mathrm{i}}$ negativas para a variável massa média de colmo. Assim, esses dois genitores estariam contribuindo com alelos favoráveis ao aumento do perfilhamento da cana-deaçúcar, produzindo progênies de menor diâmetro de colmos. Entretanto, ambos não contribuíram em elevar a $\mathrm{TCH}$ e a $\mathrm{TBH}$, pois suas estimativas de $\mathrm{g}_{\mathrm{i}}$, para essas variáveis, não diferiram estatisticamente de zero. 
Quadro 3. Estimativas da capacidade geral de combinação $\left(\mathrm{g}_{\mathrm{i}}\right)$ para número colmos (NC), massa média de colmo (MMC), Brix médio (Brix), toneladas de cana por hectare $(\mathrm{TCH})$, toneladas de Brix por hectare $(\mathrm{TBH})$ e porcentagem de florescimento $(\mathrm{F})$

\begin{tabular}{|c|c|c|c|c|c|c|}
\hline Genitores & $\mathrm{NC}$ & MMC & $\begin{array}{c}\text { Brix } \% \\
\text { caldo da cana }\end{array}$ & $\mathrm{TCH}$ & $\mathrm{TBH}$ & $\mathrm{F}$ \\
\hline & & $\mathrm{kg}$ & & \multicolumn{2}{|c|}{$\longrightarrow$ t } & $\%$ \\
\hline IAC $86-2210$ & $-23,17^{* *}$ & $0,05^{* *}$ & $0,64^{* *}$ & 0,66 & $0,68^{*}$ & $2,30^{* *}$ \\
\hline SP81-1763 & 1,53 & $-0,05^{* *}$ & 0,07 & $-4,84^{* *}$ & $-1,02^{* *}$ & $7,89^{* *}$ \\
\hline SP82-6108 & $-3,96$ & $0,17^{* *}$ & $-0,09$ & $13,54^{* *}$ & $2,92^{* *}$ & $-5,06^{* *}$ \\
\hline SP84-2029 & $11,50^{* *}$ & $-0,04^{* *}$ & $-0,15$ & $-0,90$ & $-0,35$ & $-2,41^{* *}$ \\
\hline SP86-96 & $-1,87$ & $-0,10^{* *}$ & $-0,72^{* *}$ & $-9,16^{* *}$ & $-2,68^{* *}$ & 0,17 \\
\hline SP88-754 & 1,33 & $-0,01^{* *}$ & 0,00 & 0,06 & 0,06 & $-0,80$ \\
\hline SP88-819 & $28,58^{* *}$ & $-0,05^{* *}$ & 0,12 & 2,02 & 0,53 & $-2,23^{*}$ \\
\hline SP87-365 & $-2,09$ & $-0,02^{* *}$ & 0,16 & $-2,93^{*}$ & $-0,45$ & $-0,50$ \\
\hline
\end{tabular}

$*, * *$ Significativo a $5 \%$ e $1 \%$ de probabilidade pelo teste $\mathrm{t}\left(\mathrm{H}_{0}: \mathrm{g}_{\mathrm{i}}=0\right)$ respectivamente.

Empregando também o clone SP84-2029 em seu trabalho, BRESSIANI et al. (2001) também obtiveram para esse genótipo estimativas elevadas e positivas $(p<0.05)$ de $g_{i}$ para número de colmos e não significativas para $\mathrm{TCH}$ em soca, corroborando com os resultados apresentados no quadro 3.

Os genitores SP86-96, SP88-754 e SP87-365 apresentaram estimativas dos efeitos $g_{i}$, negativas ou não diferentes estatisticamente de zero, para todas as variáveis avaliadas, ou seja, o comportamento médio desses genitores, em cruzamento com os demais, não diferiu ou foi inferior à média geral dos cruzamentos. O genitor SP81-1763 também se comportou como aqueles, porém, apresentou uma exceção que foi a estimativa de $g_{i}$ elevada e positiva para a variável porcentagem de florescimento, o que o torna ainda mais indesejável na participação em cruzamentos para o melhoramento da cana-de-açúcar. Esse fato também é evidenciado por meio do trabalho de BRESSIANI et al. (2001) em que os autores obtiveram para a variedade SP81-1763 estimativas de $g_{i}$ elevada e negativa $(\mathrm{p}<0.05)$, para a variável chochamento, evento ocasionado em sua maioria pelo florescimento.

O efeito da CEC é interpretado como o desvio de um híbrido em relação ao que seria esperado, com base CGC de seus genitores, portanto, servindo para evidenciar a importância de interações não-aditivas, resultantes da complementação gênica entre os genitores. Desse modo, o estudo da CEC permite antever a possibilidade de ganho genético com a exploração da heterose.
Altos valores, positivos ou negativos, das estimativas da capacidade específica de combinação $\left(\hat{\mathrm{s}}_{\mathrm{ij}}\right)$, demonstram que o comportamento de um cruzamento é, relativamente, melhor ou pior do que era esperado com base na CGC dos genitores.

Normalmente, interessa ao melhorista as combinações híbridas com médias elevadas, estimativas da CEC mais favoráveis, que envolvam pelo menos um dos genitores que tenha apresentado o mais favorável efeito da CGC (Cruz e Regazzi, 1994).

Com base nessas informações e nas prioridades do melhoramento da cana-de-açúcar em obter clones mais produtivos e com maior produção de açúcar por hectare, identificou-se, nos quadros 1 e 4 , que alguns cruzamentos poderiam ser úteis em suprir essas necessidades. Dentre esses se destacaram: SP82-6108 x SP88-754, SP82-6108 x SP87-365, SP811763 x SP82-6108, IAC86-2210 x SP88-754, IAC86-2210 x SP86-96 e IAC86-2210 x SP81-1763.

Verificou-se também que os cruzamentos SP88$819 \times$ SP87-365, SP84-2029 x SP87-365, SP88-754 x SP87-365, SP84-2029 x SP88-819, e SP81-1763 x SP88819 , apesar de não envolverem pelo menos um dos genitores com o mais favorável efeito da CGC, obtiveram médias elevadas para as variáveis TCH e TBH. Para os três primeiros cruzamentos mencionados, as estimativas $\hat{\mathrm{S}}_{\mathrm{ij}}$ foram significativas para as variáveis $\mathrm{TCH}$ e TBH, enquanto o quarto e o último cruzamentos apresentaram os efeitos sij significativos apenas para as variáveis TBH e TCH respectivamente. 
Quadro 4. Estimativas da capacidade específica de combinação ( $\mathrm{s}_{\mathrm{ij}}$ ) para o número de colmos (TC), massa média de colmo $(\mathrm{MMC})$, Brix médio (Brix), toneladas de cana por hectare $(\mathrm{TCH})$, toneladas de Brix por hectare $(\mathrm{TBH})$ e porcentagem de florescimento $(\mathrm{F})$

\begin{tabular}{|c|c|c|c|c|c|c|}
\hline Gruzamentos & NC & MMC & $\begin{array}{c}\text { Brix \% } \\
\text { caldo da cana }\end{array}$ & $\mathrm{TCH}$ & $\mathrm{TBH}$ & $\mathrm{F}$ \\
\hline & & $\mathrm{kg}$ & & 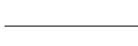 & 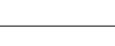 & $\%$ \\
\hline SP82-6108 x SP88-754 & 13,56 & $-0,02^{* *}$ & $-0,00$ & $8,59^{* *}$ & $1,77^{* *}$ & 0,88 \\
\hline SP82-6108 x SP87-365 & 12,70 & $0,10^{* *}$ & $0,50^{* *}$ & $9,49^{* *}$ & $2,57^{* *}$ & $4,50^{* *}$ \\
\hline SP81-1763 x SP82-6108 & $18,38^{*}$ & $0,08^{* *}$ & $-0,15$ & $8,96^{* *}$ & $1,91^{* *}$ & $-4,66^{* *}$ \\
\hline SP82-6108 x SP88-819 & $-2,53$ & $-0,01^{* *}$ & 0,22 & 0,08 & 0,46 & 1,31 \\
\hline IAC86-2210 x SP88-754 & $26,63^{* *}$ & $0,09 * *$ & $-0,27$ & $14,55^{* *}$ & $2,95^{* *}$ & $-1,03$ \\
\hline SP88-819 x SP87-365 & $32,78^{* *}$ & $0,04^{* *}$ & $-0,22$ & $14,52^{* *}$ & $2,81^{* *}$ & $-4,50^{*}$ \\
\hline IAC $86-2210 \times$ SP86-96 & 20,05 & $0,11^{* *}$ & 0,04 & $20,16^{* *}$ & $4,37^{* *}$ & 0,12 \\
\hline IAC $86-2210$ x SP81-1763 & 12,38 & $0,07^{* *}$ & $-0,20$ & $12,85^{* *}$ & $2,71^{* *}$ & $5,64^{* *}$ \\
\hline SP84-2029 x SP87-365 & 14,00 & $0,07^{* *}$ & $-0,06$ & $11,73^{* *}$ & $2,56^{* *}$ & 1,38 \\
\hline SP84-2029 x SP88-819 & $22,33^{*}$ & $-0,09 * *$ & 0,34 & 6,08 & $1,77^{*}$ & 0,65 \\
\hline SP88-754 x SP87-365 & 16,66 & $0,07^{* *}$ & 0,25 & $7,51^{*}$ & $1,94^{*}$ & 0,61 \\
\hline SP81-1763 x SP88-819 & 16,92 & $0,05^{* *}$ & $-0,14$ & $7,42^{*}$ & 1,47 & $5,51^{* *}$ \\
\hline SP82-6108 x SP86-96 & $-11,94$ & $0,02^{* *}$ & $-0,51^{* *}$ & $-0,15$ & $-0,65$ & $-1,47$ \\
\hline SP84-2029 x SP86-96 & $29,32^{* *}$ & $0,08^{* *}$ & 0,22 & $12,79^{* *}$ & $2,95^{* *}$ & 1,34 \\
\hline IAC86-2210 x SP82-6108 & $-9,70$ & $-0,11^{* *}$ & $0,30^{*}$ & $-13,13^{* *}$ & $-2,50^{* *}$ & $-0,04$ \\
\hline IAC86-2210 x SP84-2029 & 10,22 & $-0,02^{* *}$ & $-0,14$ & $-0,21$ & $-0,21$ & $-3,03^{*}$ \\
\hline SP86-96 x SP88-819 & 0,33 & $0,08^{* *}$ & $-0,62$ & 6,03 & 0,61 & 3,97 \\
\hline SP82-6108 x SP84-2029 & $-21,73^{* *}$ & $-0,08^{* *}$ & $-0,25$ & $-13,81^{* *}$ & $-3,33^{* *}$ & 1,89 \\
\hline SP84-2029 x SP88-754 & $-39,60 * *$ & $0,04^{* *}$ & 0,22 & $-5,18$ & $-0,91$ & $-1,94$ \\
\hline SP81-1763 x SP87-365 & $-4,65$ & $-0,01^{* *}$ & $-0,53^{*}$ & $-3,50$ & $-1,39$ & $1,21^{*}$ \\
\hline SP88-754 x SP88-819 & $-17,54$ & $-0,10^{* *}$ & $-0,26$ & $-15,25^{* *}$ & $-3,54^{* *}$ & 2,44 \\
\hline SP81-1763 x SP88-754 & $-15,89^{*}$ & $-0,07^{* *}$ & 0,11 & $-9,78^{* *}$ & $-2,19 * *$ & 1,63 \\
\hline SP86-96 x SP88-754 & $-4,05$ & $-0,01^{* *}$ & 0,06 & $-6,90^{* *}$ & $-1,27^{*}$ & $-0,27$ \\
\hline IAC $86-2210 \times$ SP88-819 & $-52,27^{* *}$ & $0,02^{* *}$ & $0,69^{* *}$ & $-18,88^{* *}$ & $-3,58^{* *}$ & 1,63 \\
\hline SP81-1763 x SP84-2029 & $-23,17^{* *}$ & $-0,02^{* *}$ & $-0,06$ & $-10,94^{* *}$ & $-2,41^{* *}$ & $-0,95$ \\
\hline IAC $86-2210 \times$ SP87-365 & $-23,42 * *$ & $-0,11^{* *}$ & $-0,05$ & $-14,69^{* *}$ & $-3,35^{* *}$ & $-2,41^{*}$ \\
\hline SP81-1763 x SP86-96 & 2,17 & $-0,08^{* *}$ & $0,65^{* *}$ & $-3,05$ & $-0,05$ & 0,48 \\
\hline SP86-96 x SP87-365 & $-51,36^{* *}$ & $-0,22 * *$ & $-0,26$ & $-31,58^{* *}$ & $-6,93^{* *}$ & $-4,26^{*}$ \\
\hline
\end{tabular}

*,** Significativo a $5 \%$ e $1 \%$ de probabilidade pelo teste $t\left(H_{0}: s_{i j}=0\right)$ respectivamente.

Quanto às estimativas dos efeitos $\hat{\mathrm{s}}_{\mathrm{ij}}$ para a variável porcentagem de florescimento, dentre os cruzamentos que se destacaram em elevar a TCH e a TBH, notou-se que os cruzamentos SP82-6108 x SP87365 e IAC86-2210 x SP81-1763 apresentaram valores elevados e positivos para as estimativas $\mathrm{s}_{\mathrm{ij}}$. Assim, no caso específico destas famílias, há o inconvenien- te de suas progênies tenderem a apresentar indivíduos que florescem, o que certamente aumentará o descarte de material no momento da seleção.

Entretanto, como essas famílias apresentaram médias de Brix elevadas, há possibilidade de selecionar clones de maturação precoce para cultivo no Centro-Sul do Brasil. 


\section{CONCLUSÕES}

1. Os efeitos gênicos aditivos foram predominantes na expressão das variáveis número de colmos, Brix e porcentagem de florescimento, ao passo que os efeitos gênicos não-aditivos foram mais importantes para as variáveis toneladas de cana por hectare e toneladas de Brix por hectare. Para a variável massa média de colmos, ambos os efeitos mostraram-se igualmente importantes.

2. Dentre os genitores que se destacaram, citem-se SP82-6108 e IAC86-2210. O primeiro foi importante em elevar a produtividade de cana e de açúcar, enquanto o segundo mostrou-se mais importante em aumentar a produtividade de açúcar.

3. As combinações híbridas superiores foram: SP82-6108 x SP88-754, SP82-6108 x SP87-365, SP81$1763 \times$ SP82-6108, IAC86-2210 x SP88-754, IAC86-2210 x SP86-96, IAC86-2210 x SP81-1763, SP88819 x SP87-365, SP84-2029 x SP87-365 e SP88-754 x SP87-365.

\section{AGRADECIMENTOS}

Ao CNPq e à FAPEMIG pelo suporte financeiro e pela bolsa de estudo. À Copersucar por fornecer os cruzamentos.

\section{REFERÊNCIAS BIBLIOGRÁFICAS}

BAENZIGER, P.S.; PETERSON, C.J. Genetic variation: Its origin and use for breeding self-pollinated species. In: STALKER, H.T. e MURPHY, J.P. (Eds.). Plant breeding in the 1990s. Wallingford: CAB International, 1992. p.69-100.

BARBOSA, M.H.P.; SILVEIRA, L.C.I. Metodologias de seleção, progressos e mudanças no programa de melhoramento genético da cana-de-açúcar da Universidade Federal de Viçosa, STAB, Açúcar, Álcool e Subprodutos, Piracicaba, v.18, n.3, p.3032,2000 .

BASTOS, I.T. Capacidade combinatória de clones e variedades de cana-de-açúcar (Saccharum spp.). 2000. 60f. Tese (Mestrado em Genética e Melhoramento de Plantas) - Universidade Federal de Viçosa.

BRESSIANI, J.A; BURNQUIST, W.L.; FUZATTO, S.R.; BONATO, A.L.V.; GERALDI, I.O. Combining ability in eight selected clones of sugarcane (Saccharum sp). Crop Breeding and Applied Biotechnology, Londrina, v.2, n.3, p.411-416, 2001.

CRUZ, C.D. Programa Genes: Aplicativo computacional em genética e estatística. Viçosa: Universidade Federal de Viçosa, 1997. 442 p.
CRUZ, C.D.; REGAZZI, A.J. Modelos biométricos aplicados ao melhoramento genético. Viçosa: Universidade Federal de Viçosa, 1994. 390p.

GRIFFING, B. Concept of general and specific combining ability in relation to diallell crossing systems. Australian Journal Biological Science, East Melbourne, v.9, p.463-493, 1956.

HOGARTH, D.M. Quantitative inheritance studies in sugar cane. III The effect of competition and violation of assumptions on estimation of genetic variance components. Australian Journal Research, North Brisbane, v.28, p.257-268, 1977.

HOGARTH, D.M. The effect of occidental selfing on the analysis of a diallel cross with sugarcane. Euphytica, Dordrecht, v.29, p.737-746, 1980 .

HOGARTH, D.M.; WU, K.K.; HEINZ, D.J. Estimating genetic variance in sugar cane using a factorial cross design. Crop Science, Madison, v.21, p.21-25, 1981.

HOGARTH, D.M., COX, M.C., BULL, J.K. Sugarcane improvement: Past achievements and future prospects. In: KANG, M.S. Crop Improvement for the $21^{\text {st }}$ Century. Baton Rouge: Louisiana State University, 1997. p.29-56.

KANG, M.S.; MILLER, J.D.; TAI, P.Y.P. Genetic and phenotypic path analysis and heritability in sugarcane. Crop Science, Madison, v.22, p.643-647, 1983.

LYRENE, P.M. Heritability of flowering in sugarcane. Crop Science, Madison, v.17, p.462-464, 1977.

MACHADO JUNIOR, G.R.; WALKER, D.I.; BRESSIANI, J.A.; DA SILVA, J.A.G. Emasculation of sugarcane tassels using hot water. In: INTERNATIONAL SOCIETY OF SUGAR CANE TECHNOLOGISTS CONGRESS, 22., 1995, Cartagena. Proceedings... Cali: Tecnicaña, 1995. p.346-352.

MARTINS FILHO, S.; CRUZ, C.D.; SEDIYAMA, C.S. Analysis of unbalanced diallel crosses. Revista Brasileira de Genética, Ribeirão Preto, v.15, n.4, p.853-869, 1992.

MILLER, J.D. Combining ability and yield component analyses in a five parent diallel cross in sugar cane. Crop Science, Madison, v.17, p.545-547, 1977.

REDDY, C.R.; REDDI, M.V. Degree of genetic determination, correlation and genotypic and phenotypic path analysis of cane and sugar yield in sugarcane. Indian Journal of Genetics, New Delhi, v.46, n.3, p.550-557, 1986.

SCOTT, A.J.; KNOTT, M.A. Cluster analysis method for grouping means in the analysis of variance. Biometrics, Alexandria, v.30, n.3, p.507-512, 1974.

SPRAGUE, G.F.; TATUM, L.A. General versus specific combining ability in single crosses of corn. Journal of the American Society of Agronomy, Madison, v.34, n.10, p.923-932, 1942.

YANG, T.C. AND CHU, C.C. Evaluation of combining ability in sugarcane (Part 1). Report of Taiwan Sugar Experimental Station, Tainan, v.26, p.1-10, 1962. 